\title{
Rigorous Numerics in Dynamics
}

\author{
Jan Bouwe van den Berg and Jean-Philippe Lessard
}

\section{Motivation}

Nonlinear dynamics shape the world around us, from the harmonious movements of celestial bodies, via the swirling motions in fluid flows, to the complicated biochemistry in the living cell. Mathematically these beautiful phenomena are modeled by nonlinear dynamical systems, mainly in the form of ordinary differential equations (ODEs), partial differential equations (PDEs) and delay differential equations (DDEs). The presence of nonlinearities severely complicates the mathematical analysis of these dynamical systems, and the difficulties are even greater for PDEs and DDEs, which are naturally defined on infinite-dimensional function spaces. With the availability of powerful computers and sophisticated software, numerical simulations have quickly become the primary tool to study the models. However, while the pace of progress increases, one may ask: just how reliable are our computations? Even for finite-dimensional ODEs, this question naturally arises if the system under study is chaotic, as small differences in initial conditions (such as those due to rounding errors in numerical computations) yield wildly diverging outcomes. These issues have motivated the development of the field of rigorous numerics in dynamics.

Rigorous numerics draws inspiration from the ideas in scientific computing, numerical analysis,

Jan Bouwe van den Berg is a professor in the Department of Mathematics at VU University Amsterdam. His email address is janbouwe.vanden. berg@vu.n1.

Jean-Philippe Lessard is a professor in the Department of Mathematics and Statistics at Université Laval. His email address is Jean-Phi 1 i ppe. Lessard@mat.u1ava1.ca.

For permission to reprint this article, please contact: reprintpermission@ams .org.

DOI: http://dx.doi.org/10.1090/noti1276 and approximation theory. In a nutshell, rigorous computations are mathematical theorems formulated in such a way that the assumptions can be rigorously verified on a computer. This requires an a priori setup that allows analysis and numerics to go hand in hand: the choice of function spaces, the choice of the basis functions and Galerkin projections, the analytic estimates, and the computational parameters must all work together to bound the errors due to approximation, rounding, and truncation sufficiently tightly for the verification proof to go through. The goal is to provide a mathematically rigorous statement about the validity of a concrete numerical simulation (i.e., not in some asymptotic sense where, for example, the grid size tends to zero) as interpreted as an approximate solution of the original problem. This complements the field of scientific computing, where the goal is to achieve highly reliable results for very complicated problems. In rigorous computing one is after absolutely reliable results for somewhat less complicated (but still hard) problems.

Outside dynamics, computer-assisted proofs have been used to settle famous open problems. Two prominent examples are the four-color theorem [1] and Kepler's densest sphere packing problem [2]. In dynamical systems, an early success is the demonstration of the universality of the Feigenbaum constant [3]. More recently, rigorous numerics were used to prove the existence of the strange attractor in the Lorenz system, which seemed, for decades, tentatively intuitive from computer simulations [4]. This settled the fourteenth problem in Smale's list of problems for the twenty-first century (the only other problem from the list that has been solved is the Poincaré conjecture). 
Computers have long played a pivotal role in the study of dynamical systems. Starting from the very first glimpses of the Mandelbrot set, computer simulations have provided a way to delve deeply into the complex behavior of nonlinear dynamics. Nevertheless, the field of dynamical systems is not dominated by computers. Quite the opposite-the theory of dynamical systems is a thriving area of mathematics, as exemplified by several recent Fields Medals. While the strength of analytic results in dynamical systems lies in characterizing generic behavior, i.e., outlining what one should typically expect "on average" in classes of systems, it is very difficult to check that any specific system is sufficiently "unexceptional" to be described by these general results. It is precisely this weakness of the general analytic theory that is the strength of computer-assisted approaches. This is of importance, since in applications one is usually interested in the behavior of a specific system. Moreover, while mathematical analysis is strong on general "existence" theorems for families of problems, information about the shape of the solutions (e.g., the patterns they describe) can usually be obtained only with the help of computer calculations (rigorous or not). While in applications one needs to be sure that a solution exists, it is usually essential to know what the solution looks like as well.

From a mathematical point of view, the advantage of rigorously validated computations over simulations is that the outcomes can be used as components in the "building" of mathematics. This is often expressed in the form of forcing theorems: if one finds a certain type of solution, then this implies, by analytic theory, many other properties of the dynamical system. The most famous result of this type is "period-3 implies chaos" for interval maps [5]. Other such examples leading to chaos are given by the existence of a Shilnikov bifurcation [6] or the existence of a single braided periodic orbit [7]. Mathematics in general is riddled with such statements, where the assumptions in the theorems are in practice impossible to check for any specific system, at least by hand. It is in overcoming this obstacle that computer-assisted proofs are at their best.

\section{Strategy}

Let us sketch the strategy for finding a solution in a nonlinear dynamical system via a computerassisted proof. We are looking for a "dynamically invariant object," which we denote abstractly by $x$ and which may be an equilibrium, a periodic or connecting orbit, or more generally an invariant manifold. Having identified a formulation for such a problem of the form $f(x)=0$ that is suitable for the analysis to follow, our starting point is a numerically obtained approximation, $x_{\text {approx }}$, of a zero of $f$, i.e., $f\left(x_{\text {approx }}\right) \approx 0$. Next, we exploit a common strategy in mathematical analysis, namely, we turn the problem $f(x)=0$ into an equivalent fixed point problem. Instead of trying to solve $f(x)=0$, we consider a map $T$ whose fixed points are the zeros of $f$. The choice of the map $T$ is not straightforward, but often an approximate Newton scheme of the form $T(x)=x-A f(x)$ is suitable, where the linear operator $A$ is some cleverly chosen approximation of the inverse of $D f(x)$. The inverse of the Jacobian itself is usually too complicated to use directly.

We then set out to prove that $T$ is a contraction on a neighborhood of $x_{\text {approx. }}$. Although this may seem a rather trivial reformulation, the essential advantage is that instead of trying to prove equalities in the formulation $f(x)=0$, contractivity involves inequalities only. This provides the flexibility ("room to play with") that is so typical of many arguments in analysis. In particular, in the context of computer-assisted proofs, inequalities allow the control of errors from a variety of sources: rounding of floating point numbers, finite-dimensional truncation (discretization of a continuous problem), "modeling" error (e.g., using a Taylor polynomial for modeling a nonlinearity rather than the Taylor series), as well as uncertainties in parameter values.

In this approach to computer-assisted proofs it is not the computer which does all the work. On the contrary: the hard work, by pencil and paper, is to reduce the problem to checking finitely many inequalities. This involves the analytic study of the defect $T\left(x_{\text {approx }}\right)-x_{\text {approx }}$ as well as the derivative of $T$ near $x_{\text {approx }}$. The proof then proceeds by a Newton-Kantorovich type argument to find a small ball $B$ around $x_{\text {approx }}$ on which the map $T$ is contracting.

The first central difficulty lies in analytically quantifying "how nonlinear" the map is. In that sense, the hurdle is essentially the same as for purely analytic techniques, where one also needs to control nonlinear and/or off-diagonal terms by (functional analytic) estimates. The second fundamental issue mirrors the situation in numerical analysis and scientific computing, namely, estimating the "cut-off error" caused by projecting the infinite-dimensional problem onto a finite-dimensional computational space (the error induced by truncating the continuous problem to a discrete one). Roughly, one needs to choose a well-adapted basis and/or a good preconditioner to obtain good estimates.

We note that all the obtained bounds need to be explicit and sufficiently sharp to be able to check, in the final step of the proof, that the inequalities guaranteeing contractivity of $T$ hold. In principle the inequalities could be checked by hand, but in practice they involve too many terms to make that feasible. Moreover, the expressions for the inequalities involve the set of floating point numbers 
$x_{\text {approx }}$, since the ball $B$ is centered at a numerically determined point. This is a crucial difference from conventional analytic results, where the ball is typically centered around some relatively simple, asymptotic limit case that is amenable to "regular" analysis.

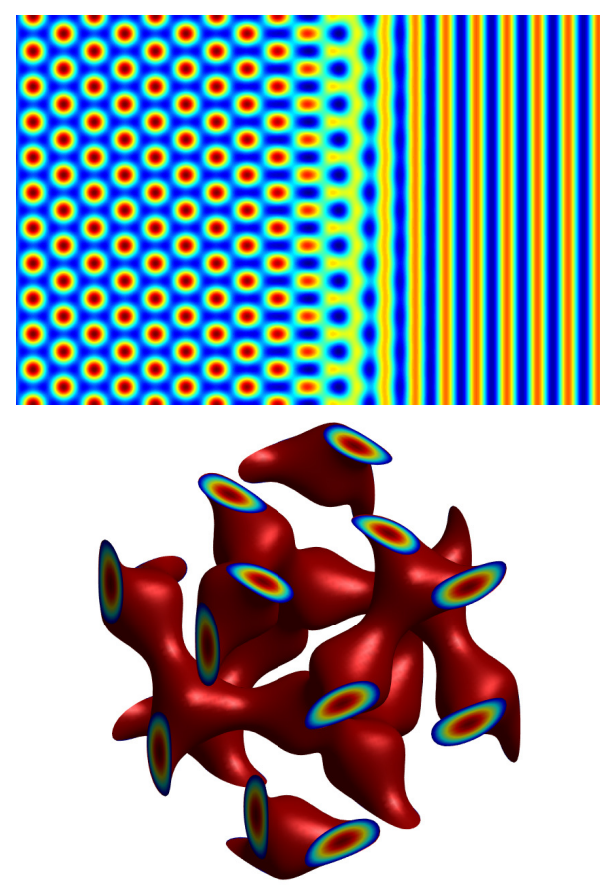

Figure 1. Top: Stationary coexistence of hexagons (spots) and rolls (stripes) of (2). Bottom: A double gyroid solution of (3) for parameter values $\mu=0.1$ and $\gamma=2.1$. Images courtesy of Jan Bouwe van den Berg.

Additionally, in the computer-assisted context, the definition of the linear operator $A$, which is a constituent of the map $T$, involves the numerical Jacobian of the truncated problem at $x_{\text {approx }}$, which is another source of floating point numbers in the inequalities to be checked. Having done the hard work in the analysis of reducing the problem to finitely many explicit inequalities, one therefore resorts to interval arithmetic computer calculations for this final step of the proof.

The above outline shows that the arguments are "quasi-analytic": the majority of the analysis is done by hand, followed by a final check of a finite list of inequalities through a computer calculation. This means that we can easily deal with parameters in the problem. Indeed, parameters play an important role in virtually all nonlinear dynamical systems that appear in applications. Therefore, rigorous parameter continuation is a vital tool in most applications, and this is incorporated in the computer-assisted approach in a relatively straightforward manner [8], [9]. Finally, we remark that contractivity implies not just existence and uniqueness but also robustness with respect to small variations in parameters. In a dynamical systems context this can be made precise in terms of hyperbolicity or transversality of the solution. Such a robustness ("no fluke") property, which is usually required to derive forcing results, is automatic from the contractivity of the operator $T$.

\section{Applications}

Techniques for computer-assisted proofs in $\mathrm{dy}$ namics are rapidly developing, and some of them, at least when applied to systems of ODEs, are becoming "routine" and are implemented in software packages such as CAPD [10]. Using such software or the ideas mentioned in the previous section, one can obtain computer-assisted proofs of existence of bounded solutions such as equilibria, periodic orbits, and connecting orbits. Bifurcation points, stable and unstable manifolds of equilibria and periodic orbits, and existence of chaos in the form of symbolic dynamics can also be studied rigorously for finite-dimensional nonlinear dynamical systems. More recently, infinite-dimensional nonlinear problems have been studied via computer-assisted proofs. Equilibria of PDEs [11], [12], [13], periodic orbits of PDEs [14], [15], solutions of boundary value problems [16], and traveling waves [17] have all been proved with the techniques of rigorous numerics. Rather than presenting an extensive list of results in the field, we choose to briefly present three sample results, and we refer to [18], [19], [20] for a more thorough discussion of applications to finite- and infinite-dimensional problems.

a) An old conjecture in delay equations. In 1955 , E. M. Wright considered the equation

(1) $y^{\prime}(t)=-\alpha y(t-1)[1+y(t)], \alpha>0$,

because of its role in the distribution of prime numbers [21]. A conjecture (stated by Jones in 1962 [22]) asserts that (1) has a unique slowly oscillating periodic solution (SOPS) for all $\alpha>\pi / 2$, i.e., a periodic solution that oscillates around 0 , spending more than one unit of time (per period) on either side of 0 . With the help of Fourier series, a rigorous parameter continuation of the SOPS was performed in [23] using the ideas of the previous section, yielding substantial progress toward the proof of the conjecture.

b) Coexistence of patterns in a PDE model. The ideas of rigorous numerics were applied in [24] to prove existence of standing waves between rolls and hexagonal patterns of the two-dimensional pattern formation PDE model

$$
\begin{aligned}
\partial_{t} u & =-(1+\Delta)^{2} u+\mu u-\beta|\nabla u|^{2}-u^{3}, \\
u & =u(x, t) \in \mathbb{R}, x \in \mathbb{R}^{2}, t \geq 0,
\end{aligned}
$$

for small parameter values $\mu, \beta \in \mathbb{R}$ (see top of Figure 1). Using the weakly nonlinear analysis of [25] proving coexistence of the patterns reduces to proving existence of heteroclinic solutions 
in a system of second-order nonlinear ODEs. After reformulating the problem as a projected boundary value problem (BVP) with boundaries in the stable/unstable manifolds, the techniques of the previous section were used to compute the local manifolds and to solve the BVP using Chebyshev series.

c) Steady states of the Ohta-Kawasaki problem. The Ohta-Kawasaki equation

$$
\partial_{t} u=-\Delta\left(\gamma^{-2} \Delta u+u-u^{3}\right)-(u-\mu)
$$

models the evolution of di-block copolymers [26], [27]. Depending on the value of the parameters $\mu$ and $\gamma$, which represent a measure of the ratio of the mixture of the polymers and the incompatibility of the polymer types, respectively, there is a multitude of stationary states with a truly three-dimensional geometry. These have been studied using the rigorous numerical techniques described above (see [28]), and we depict one rigorously verified equilibrium pattern, called a double gyroid, in Figure 1 on the bottom.

\section{Future Goals}

The past decade has seen enormous advances in the development of rigorously verified computing with the most significant results for finite-dimensional systems. While encouraging first steps for infinitedimensional systems are starting to appear, many interesting future directions remain to be explored. For instance, developing rigorous computational tools to study global dynamics of PDEs, finding bounded invariant sets for state-dependent delay equations, and demonstrating chaos in infinitedimensional continuous dynamical systems are some of the main challenges in the field. Aiming to understand global properties of dynamical systems, combining rigorous numerics with topological methods such as Morse theory, is the subject of active research. Important contributions in that direction are currently being developed based on Morse-Conley theory [29].

Finally, it is a nontrivial problem to make sure that all steps in the process (including the code) are correct. As the code for the computer-assisted proofs is getting more and more complicated, the possibilities for human error while developing the necessary analytic estimates and while implementing the algorithms are increasing. From that point of view, mathematics in the era of computers calls for the development of automatic proof assistants; see e.g., [30].

\section{References}

[1] Neil Robertson, DANiel SANDers, Paul Seymour, and RoBin ThOMAS, The four-colour theorem, $J$. Combin. Theory Ser. B, 70(1):2-44, 1997.

[2] Thomas C. Hales, A proof of the Kepler conjecture, Ann. of Math. (2), 162(3):1065-1185, 2005.
[3] OSCAR E. LANFORD III, A computer-assisted proof of the Feigenbaum conjectures, Bull. Amer. Math. Soc. (N.S.), 6(3):427-434, 1982.

[4] WARWICK TUCKER, A rigorous ODE solver and Smale's 14th problem, Foundations of Computational Mathematics, 2(1):53-117-117, 2002-12-21.

[5] Tien Yien Li and James A. Yorke, Period three implies chaos, Amer. Math. Monthly, 82(10):985-992, 1975.

[6] L. P. SHIL'NIKOV, A case of the existence of a denumerable set of periodic motions, Dokl. Akad. Nauk SSSR, 160:558-561, 1965.

[7] JAN BOUWE VAN DEN BERG and JEAN-PHILIPPE LESSARD, Chaotic braided solutions via rigorous numerics: Chaos in the Swift-Hohenberg equation, SIAM J. Appl. Dyn. Syst., 7(3):988-1031, 2008.

[8] JAN BOUWE VAN DEN BERG, JEAN-PHILIPPE LESSARD, and KONSTANTIN MISCHAIKOW, Global smooth solution curves using rigorous branch following, Math. Comp., 79(271):1565-1584, 2010.

[9] MARCiO GAMEIRO, JEAN-Philippe LESSARD, and ALESSANDRO PUgLIESE, Computation of smooth manifolds of solutions of PDEs via rigorous multiparameter continuation, Found. Comput. Math., 2015.

[10] CAPD: Computer assisted proofs in dynamics, a package for rigorous numerics, capd. i i.uj.edu.p1/.

[11] PIOTR ZgLiCZYŃSKI and KONSTANTIN MisCHAIKOW, Rigorous numerics for partial differential equations: The Kuramoto-Sivashinsky equation, Found. Comput. Math., 1(3):255-288, 2001.

[12] MYOUnGNYOUN Kim, MitsuHiRo T. NAKAO, YoshiTAKA WATANABE, and TAKAAKI NISHIDA, A numerical verification method of bifurcating solutions for 3dimensional Rayleigh-Bénard problems, Numer. Math. 111(3):389-406, 2009.

[13] MARCio GAMEIRo and JeAN-Philippe Lessard, Analytic estimates and rigorous continuation for equilibria of higher-dimensional PDEs, J. Differential Equations, 249(9):2237-2268, 2010.

[14] GIANNI ARIOLI and HANS KocH, Integration of dissipative partial differential equations: A case study, SIAM J. Appl. Dyn. Syst., 9(3):1119-1133, 2010.

[15] PIOTR ZgLICZYŃSKI, Rigorous numerics for dissipative partial differential equations, II. Periodic orbit for the Kuramoto-Sivashinsky PDE-a computer-assisted proof, Found. Comput. Math., 4(2):157-185, 2004.

[16] B. Breuer, P. J. McKenna, and M. Plum, Multiple solutions for a semilinear boundary value problem: A computational multiplicity proof, J. Differential Equations, 195(1):243-269, 2003.

[17] B. Breuer, J. Horák, P. J. McKenna, and M. Plum, A computer-assisted existence and multiplicity proof for travelling waves in a nonlinearly supported beam, J. Differential Equations, 224(1):60-97, 2006.

[18] WARWICK TUCKER, Validated Numerics, A Short Introduction to Rigorous Computations, Princeton University Press, Princeton, NJ, 2011.

[19] SIEGFRIED M. RUMP, Verification methods: Rigorous results using floating-point arithmetic, Acta Numer., 19:287-449, 2010.

[20] M. T. NAKAO, Numerical verification methods for solutions of ordinary and partial differential equations, Numer. Funct. Anal. Optim., 22(3-4):321-356, 2001.

[21] E. M. Wright, A non-linear difference-differential equation, J. Reine Angew. Math., 194:66-87, 1955. 
[22] G. STEPHeN Jones, On the nonlinear differentialdifference equation $f^{\prime}(x)=-\alpha f(x-1)\{1+f(x)\}$, J. Math. Anal. Appl., 4:440-469, 1962.

[23] JEAN-PHILIPPE LESSARD, Recent advances about the uniqueness of the slowly oscillating periodic solutions of Wright's equation, J. Differential Equations, 248(5):992-1016, 2010.

[24] JAN BOUWE VAN DEN BERG, ANDRÉA DESCHÊNES, JEAN-PHILIPPE LESSARD, and JASON MiRELES JAMES, Stationary coexistence of hexagons and rolls via rigorous computations, SIAM J. Appl. Dyn. Syst., 14(2):942-979, 2015.

[25] ARJen DoElman, BJöRn SANDSTEDE, ARND SCHEel, and GUIDO SCHNEIDER, Propagation of hexagonal patterns near onset, European J. Appl. Math., 14(1):85-110, 2003.

[26] TAKAO OHTA and KYOZI KAWASAKI, Equilibrium morphology of block copolymer melts, Macromolecules, 19(10):2621-2632, 1986.

[27] M. BAHIANA and Y. OONO, Cell dynamical system approach to block copolymers, Phys. Rev. A, 41:6763-6771, Jun 1990.

[28] JAN BOUWE VAN DEN BERG and J. F. Williams, Rigorous numerics for the Ohta-Kawasaki problem, in preparation, 2015.

[29] Zin Arai, William Kalies, Hiroshi KoKubu, KonSTANTIN Mischaikow, Hiroe OKA, and PAWEe PILARCZYK, A database schema for the analysis of global dynamics of multiparameter systems, SIAM J. Appl. Dyn. Syst., 8(3):757-789, 2009.

[30] The Coq Proof Assistant, https://coq.inria.fr.

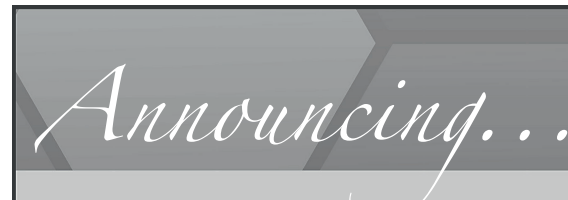

The creators of MathJobs.Org welcome you to: MathPrograms.Org

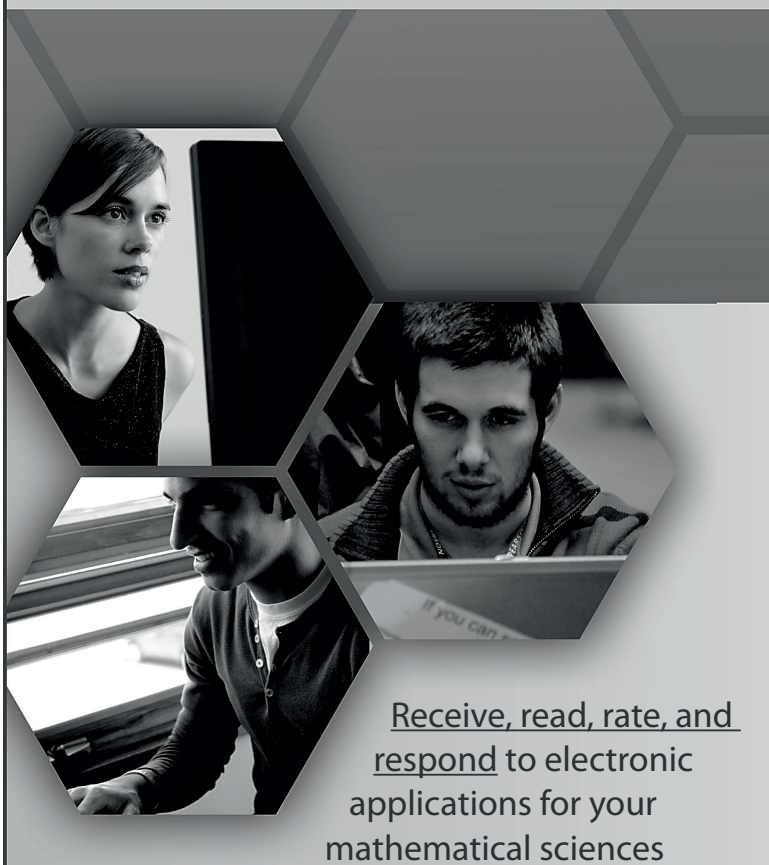

programs, such as undergraduate summer research programs and travel grant competitions.

Customize your settings and control the application form; also set secure access for the admissions committee.

Enter program announcements for public display.

Download data to personal computers for use in word processing and spreadsheets or as a full permanent storage file.

Service is FREE to applicants. Institutions pay annually for one program or for multiple programs. 